\title{
Changes in Pigment Concentrations Associated with the Degree of Sunburn Browning of 'Fuji' Apple
}

\author{
David A. Felicetti and Larry E. Schrader ${ }^{1}$ \\ Washington State University Tree Fruit Research and Extension Center, 1100 N. Western Avenue, \\ Wenatchee, WA 98801
}

\begin{abstract}
AdDitional INDEX words. Malus domestica, chlorophyll, carotenoid, anthocyanin, quercetin, HPLC
Abstract. Significant changes in pigments were identified, quantified, and correlated to the changes in color associated with sunburn browning (SB) of 'Fuji' apples (Malus domestica Borkh.). Apples were sorted into five classes: NB, no sunburn; SB-1 to SB-4, increasing severity of sunburn browning. A decline in chlorophylls a and $b$ and reduced anthocyanin accumulation with increased sunburn severity were observed. A significant increase in total quercetin glycosides was seen with slight sunburn (i.e., SB-1) with most of the increase resulting from increased quercetin 3galactoside and quercetin 3-glucoside + quercetin 3-rutinoside. Quercetin glycosides increased modestly from SB-1 to SB-4, but few differences were statistically significant. $\beta$-carotene increased in sunburned apples both years, but changes in SB-3 and SB-4 were inconsistent between the 2 years. The xanthophylls were significantly higher $(P<0.05)$ in SB-1 than in NB, but no difference was detected from SB-1 to SB-4. Lutein, a xanthophyll, showed no change as a result of sunburn in either year. Hue angle was highly correlated $(P<0.05)$ to the concentrations of total chlorophylls, idaein, and total quercetin glycosides. Despite minor differences between growing seasons, the overall trends of decreased chlorophyll and idaein, and increased quercetin glycosides and carotenoids persisted. Lower chlorophyll and anthocyanin concentrations observed in the sunburned apples allowed the yellows from the carotenoids and quercetin glycosides to be more prominent. Additionally, the increases in carotenoid and quercetin glycoside concentrations of sunburned apples made the change in color more striking.
\end{abstract}

Apple sunburn is a serious problem in many parts of the world where apples are grown in the presence of high solar irradiance and high temperature. Three types of apple sunburn have been characterized in the author's laboratory at Washington State University. Sunburn necrosis and sunburn browning were described by Schrader et al. (2001, 2003a, b), and photoxidative sunburn was first described by Felicetti (2003). Sunburn necrosis occurred when fruit surface temperatures (FSTs) reached $52 \pm 1{ }^{\circ} \mathrm{C}$, was the result of heat-induced cell death, and did not require direct sunlight (other than as a heat source). Sunburn necrosis appeared on the surface of the apple as a dark-brown or black spot several millimeters thick. The second type of sunburn, sunburn browning, did not result in cell death and was characterized by a yellowing or browning of the skin. It occurred at lower FSTs $\left(46\right.$ to $49^{\circ} \mathrm{C}$ ) than those required for sunburn necrosis and required sunlight (Schrader et al., 2001, 2003b). The minimum FST needed for sunburn browning was referred to as the threshold temperature (Schrader et al., 2001). The threshold temperature was specifically defined as the minimum FST needed to induce sunburn browning when fruit was exposed to sunlight for $1 \mathrm{~h}$ (Schrader et al., 2001). The third type of sunburn is photoxidative sunburn, which has been hypothesized to be a purely photoxidative process that results in photobleaching of shaded peel suddenly exposed to sunlight (Felicetti, 2003).

Schrader et al. (2003a) classified the degree of apple sunburn into six classes based on appearance. In this system, a rating of

Received for publication 27 June 2007. Accepted for publication 5 Oct. 2007. This paper is a portion of a dissertation submitted by the senior author in partial fulfillment of the requirements for the degree of Doctor of Philosophy in Horticulture.

We gratefully acknowledge financial support from the Washington Tree Fruit Res. Commission. We also thank James Mattheis (USDA/ARS) for the use of his high-performance liquid chromatography system.

${ }^{1}$ Corresponding author. E-mail: schrader@wsu.edu.
0 denotes no sunburn, a rating of 5 signifies sunburn necrosis, and ratings of 1 to 4 refer to different degrees of sunburn browning. In red cultivars, degrees of sunburn browning (1-4) were based on reduced red color and increasing yellow and brown colors. In all cultivars, increased sunburned area, increased browning, and increased blackened lenticels in the affected area were observed as the degree of sunburn browning increased (i.e., 1 had more red color, less browning, fewer blackened lenticels, and a smaller affected area than 2 to 4 ). Schrader et al. (2003a) implied that degrees 1 to 4 were the result of increased exposure to sunburn browning induction factors (e.g., sunlight and heat).

This study focused on 'Fuji' sunburn degrees 0 to 4, with rating 5 omitted because it is a different type of sunburn. 'Fuji' apples were chosen for this study because they are economically important to Washington state, they show moderate sunburn susceptibility in the Pacific northwestern United States, and they contain anthocyanins. The intent of this study was to characterize the changes in pigments in an attempt to understand better how high solar radiation and temperature affect apple fruit and to determine which changes in pigment concentrations were related to the observed color changes associated with sunburn.

\section{Materials and Methods}

Site AND Plant material. This study was conducted in the 2005 and 2006 growing seasons on 'Fuji' apples. In 2005, the orchard was located in Wenatchee, WA, at the Washington State University-Tree Fruit Research and Extension Center. In 2006, a commercial orchard west of Yakima, WA, was used. Both orchards received standard horticultural practices and disease and pest control (Smith, 2007). Apples were collected on 14 Oct. 2005 and 18 Oct. 2006 from their respective 
orchards, placed in regular atmosphere cold storage at $0.5^{\circ} \mathrm{C}$, and sampled the following day. Classification used to separate sunburn browning into classes was modified from Schrader et al. (2003a). The highest classification (rating 5) was eliminated, the nonsunburned apple was labeled NB, and increasing severity of sunburn browning was labeled SB-1, SB-2, SB-3, and SB-4 respectively (Fig. 1).

SAMPLING. In 2005, 10 apples of sunburn degrees 0 to 4 were selected and four peel disks ( $16 \mathrm{~mm}$ in diameter, $1 \mathrm{~mm}$ thick) were taken from each apple. Two disks were used for chlorophyll and carotenoid analysis, and two disks were used for phenolic analysis. Thus, each degree (class) of sunburn had 10 repetitions, with each repetition composed of two peel disks from the same apple. In 2006, 20 apples of sunburn degrees 0 to 4 were selected and two peel disks $(12 \mathrm{~mm}$ in diameter, $1 \mathrm{~mm}$ thick) were taken from the sun-exposed side of each apple. One of the disks was for chlorophyll and carotenoid analyses, and the other was for phenolic analysis. Four disks from four different apples were pooled to make five repetitions per degree of sunburn, with each repetition composed of four disks from four apples. All samples were immediately frozen in liquid nitrogen and stored at $-80{ }^{\circ} \mathrm{C}$ until analyses were performed.

Peel color analyses. Color of the area to be sampled on the sun-exposed side of each apple was determined using a colorimeter (CR-300 Chroma Meter; Minolta Corp., Osaka, Japan). The CIE L*a*b* (L*, lightness coordinate; $a^{*}$, red/ green coordinate; $\mathrm{b}^{*}$, yellow/blue coordinate) color space was used and the hue angle $\left[\mathrm{h}^{\circ}, \tan ^{-1}\left(\mathrm{~b}^{*} / \mathrm{a}^{*}\right)\right]$ and chroma $\left[\mathrm{C}^{*}, \sqrt{\left(a^{*}\right)^{2}+\left(b^{*}\right)^{2}}\right]$ were calculated (McGuire, 1992).

Extractions. Frozen samples were crushed and ground into a fine powder using a mortar and pestle, and liquid nitrogen was added immediately before the extractions because some of the pigments are unstable. All extracts were filtered $(0.45-\mu \mathrm{m}$ PTFE membrane) into amber vials. Light exposure was minimized throughout the extraction process by performing extractions in near-dark conditions in a hood, with only indirect light from an adjoining laboratory. Extracts were kept in the dark when not being handled.

ChLOROPHYLL AND CAROTENOID EXTRACTIONS. The extraction method used was modified from Rudell et al. (2002). We experienced the same chlorophyll a (chl a) degradation that prevented them from quantifying chl a using high-performance liquid chromatography (HPLC) and determined that the degradation was likely the result of acid hydrolysis (data not shown). As a result, a buffer was incorporated into the extraction as follows: Buffer $(0.5 \mathrm{~mL}$; $0.1 \mathrm{~m}$ HEPES, adjusted to $\mathrm{pH} 7$ using $0.5 \mathrm{~m} \mathrm{KOH}$ ) was added followed by $1 \mathrm{~mL} 100 \%$ acetone, and the tissue was ground for an additional $1 \mathrm{~min}$. The



Fig. 1. Degrees of sunburn browning of 'Fuji' apples as modified from Schrader et al. (2003a). NB, no sunburn; SB-1, sunburn severity 1; SB-2, sunburn severity 2; SB-3, sunburn severity 3; SB-4, sunburn severity 4. resultant homogenate was centrifuged at $12,000 \mathrm{~g}_{\mathrm{n}}$ for $2 \mathrm{~min}$. Mortar and pestle were rinsed thrice with $1 \mathrm{~mL} 100 \%$ acetone, and the rinsate was used to resuspend the pellet. The resultant suspension was centrifuged and the supernatants were combined and partitioned into hexanes $(3 \times 1 \mathrm{~mL})$. The hexanes phase was dried using a rotoevaporator (vacuum of $101.3 \mathrm{kPa}$; water bath at $20^{\circ} \mathrm{C}$ ) and reconstituted in $100 \mu \mathrm{L} 100 \%$ acetone.

Phenolic extractions. The phenolic extraction was modified from Rudell et al. (2002) as follows: One milliliter of acidified methanol $(1 \% \mathrm{HCl} \mathrm{v} / \mathrm{v})$ was added to the mortar and the tissue was ground for an additional $1 \mathrm{~min}$. The homogenate was centrifuged at $12,000 g_{\mathrm{n}}$ for $2 \mathrm{~min}$. The mortar and pestle were washed with acidified methanol $(3 \times 1 \mathrm{~mL})$, and washes were used to resuspend the pellet. This suspension was centrifuged for $2 \mathrm{~min}$ and the supernatant was combined with the first. The combined supernatants were washed with $1 \times 3$ $\mathrm{mL}$ hexanes, and the hexanes layers were discarded. The aqueous phase was rotoevaporated under a vacuum of 101.3 $\mathrm{kPa}$ to concentrate phenolics and remove any residual hexanes.

Pigment analyses. Twenty-five-microliter samples were analyzed using a reverse-phase HPLC system equipped with a Series 1100 Hewlett Packard injector, a Hypersil ODS guard column $(5 \mu \mathrm{m}, 4.0 \times 4 \mathrm{~mm}$; Agilent Technologies, Santa Clara, CA), a Hypersil ODS column $(5 \mu \mathrm{m}, 4.0 \times 125 \mathrm{~mm}$; Agilent Technologies), and a photodiode array detector (model 996; Waters, Milford, MA). Pigments were eluted at a flow rate of $1 \mathrm{~mL} \cdot \mathrm{min}^{-1}$ and a column temperature of $25^{\circ} \mathrm{C}$ using a binary gradient.

The chlorophyll and carotenoid gradient used was modified from Rudell et al. (2002) and consisted of $100 \%$ solvent A [80:20 Methanol-0.1 м HEPES buffer ( $\mathrm{pH}$ 7.0, titrated with 0.5 M KOH) (v/v)] for the first 2 min, then solvent B (ethyl acetate) increased linearly and reached $50 \%$ at $21 \mathrm{~min}$. This mixture was maintained until $40 \mathrm{~min}$, at which time the column was reequilibrated with $100 \%$ solvent $\mathrm{A}$ for $12 \mathrm{~min}$. The $0.1 \mathrm{M}$ HEPES buffer was adjusted to $\mathrm{pH} 7.0$ before mixing with methanol.

The phenolic gradient used was modified from Rudell et al. (2002) and consisted of $100 \%$ solvent $\mathrm{A}\left(1: 10: 89 \mathrm{H}_{3} \mathrm{PO}_{4^{-}}\right.$ methanol-deionized water $\mathrm{v} / \mathrm{v}$ ) for the first $2 \mathrm{~min}$ and then decreased linearly to $20 \%$ solvent A and $80 \%$ solvent B (1:70:29 $\mathrm{H}_{3} \mathrm{PO}_{4}$-methanol-deionized water $\left.\mathrm{v} / \mathrm{v}\right)$ at $36 \mathrm{~min}$. From 36 to $40 \mathrm{~min}$, solvent B increased linearly to $100 \%$. The run ended at $40 \mathrm{~min}$, at which time the column was reequilibrated with $100 \%$ solvent $\mathrm{A}$ for $13 \mathrm{~min}$.

Peak identification and Quantification. Peaks were identified by retention time and spectral comparison with authentic standards. Peaks were quantified using molar absorption coefficients derived from authentic standards. Exceptions were violaxanthin and antheraxanthin, which were identified based on the elution order and relative peak heights of similar HPLC methods (Thayer and Bjorkman, 1990; Yamauchi and Watada, 1991). The lutein molar extinction coefficient was used to quantify violaxanthin and antheraxanthin. Quercetin 3-xyloside, quercetin 3-arabinopentoside, and quercetin 3-arabinofuranoside were tentatively identified based on their retention times, ultraviolet/visible 
spectra, elution order, and their presence in apple as reported previously (Rudell et al., 2002; Schieber et al., 2002). The xanthophylls and $\beta$-carotene were quantified at $446 \mathrm{~nm}$. Chlorophyll b (chl b) was quantified at $466 \mathrm{~nm}$ and chl a at $660 \mathrm{~nm}$. Epicatechin, chlorogenic acid, the quercetin glycosides, and idaein were quantified at $280 \mathrm{~nm}, 328 \mathrm{~nm}, 357 \mathrm{~nm}$, and $519 \mathrm{~nm}$ respectively. Lutein, $\beta$-carotene, chl a, chl b, quercetin 3- $\beta$-D-glucoside, quercetin rutinoside, quercetin 3-Dgalactoside, quercetin 3-L rhamnoside, epicatechin, and chlorogenic acid were purchased from Sigma-Aldrich Co. (St. Louis).

Statistical analyses. One-way analysis of variance was performed to determine whether significant differences existed among the pigment concentrations of the peel types. Fisher's (LSD) was calculated when appropriate to determine which means were statistically different $(P<0.05$, Proc GLM, Means/ LSD; SAS Institute, Cary, NC). The hue angle was regressed against the concentrations of total chlorophylls, total carotenoids, total quercetin glycosides, and idaein to determine linear $R^{2}$ (Proc REG; SAS Institute).

\section{Results}

Both years, chl a and chl b concentrations decreased with increased severity of sunburn (Fig. 2). In 2005, the chl a concentration in NB apples was significantly higher than in SB-
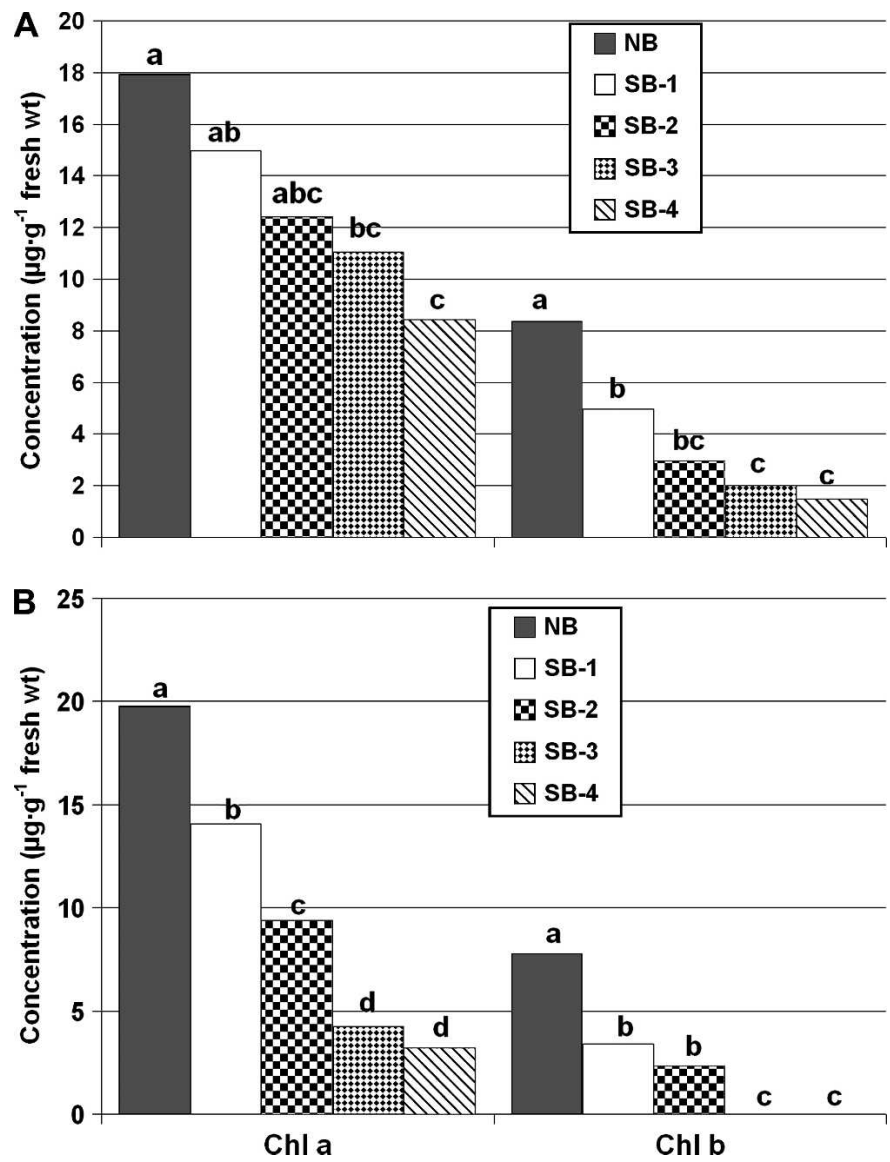

Fig. 2. (A, B) Chlorophyll a (Chl a) and chlorophyll b (Chl b) concentrations in micrograms per gram fresh weight for 'Fuji' apple in 2005 (A) and 2006 (B) with peel types NB (no sunburn), SB-1, SB-2, SB-3, and SB-4 (sunburn severity 1-4 respectively). Within pigment and year, bars with the same letter are not significantly different via LSD at $P \leq 0.05$.
3 and SB-4, whereas chl b concentrations in SB-1 to SB-4 were significantly lower than in NB. In 2006, the concentration of chl $a$ and chl $b$ in SB-1 to SB-4 were significantly lower than in NB, with SB-3 and SB-4 being significantly lower than SB-1 and SB-2. In 2006, there was no detectable chl b in SB-3 and SB-4. Hence, the decrease in chl b was not proportional to the decrease in chl a, as is demonstrated by a marked increase in chl a-to-chl b ratios with increased sunburn severity (Table 1).

Both years, xanthophyll results were consistent (Fig. 3). Violaxanthin, antheraxanthin, and total xanthophylls in burned apples were higher than in NB apples, but little difference was observed among burned apples (SB-1 to SB4). In 2005, there were no significant differences in lutein concentrations. In 2006, lutein in SB-3 and SB-4 was significantly lower than in SB-1 and SB-2. $\beta$-carotene was inconsistent between the 2 years. In 2005, SB-3 was no different from NB, but was lower than SB-2 and SB-4. In 2006, this was not seen, but a substantial increase in $\beta$-carotene was determined in SB-4.

Idaein is the only anthocyanin reported here because it is the main anthocyanin in apple. Other anthocyanins previously reported in apple are not reported in this study because their peaks could not be quantified consistently. Idaein concentrations decreased with increased sunburn severity (Fig. 4). In 2005, idaein concentration steadily decreased with sunburn severity, but SB-1 was not different from NB. In 2006, the decrease was very large between NB and SB-1, after which modest decreases were detected. In both years the idaein concentrations of SB-4 were lower than in SB-3, but the differences were not significant.

Quercetin glycosides increased consistently both years (Fig. 5). The main glycosides, quercetin 3-galactoside and quercetin 3-glucoside (Glu + Rut in Fig. 5), were dramatically higher in the SB-1 when compared with the NB samples and showed increases of $116 \%$ and $307 \%$ in 2005 and $69 \%$ and $183 \%$ in 2006 respectively. More modest increases in these two quercetin glycosides were detected from SB-1 to SB-4. Quercetin 3 -xyloside, quercetin 3-arabinopyranoside, quercetin 3-arabinofuranoside, and quercetin 3-rhamnoside increased more modestly over most peel types. Concentrations of quercetin, the aglycone of the quercetin glycosides, are reported here even though it is believed that no free quercetin is stored in apple peel. Based on a separate experiment (Table 2), quercetin was

Table 1. Chlorophyll a/b ratios for NB (no sunburn), SB-1, SB-2, SB-3, and SB-4 (sunburn severity 1-4 respectively) 'Fuji' apple peel types in 2005 and 2006.

\begin{tabular}{lll}
\hline & \multicolumn{2}{c}{ Chlorophyll a/b (ratio) } \\
\cline { 2 - 3 } Peel type & 2005 & 2006 \\
\hline NB & 2.19 & 2.55 \\
SB-1 & 3.23 & 4.08 \\
SB-2 & $4.07^{z}$ & 4.06 \\
SB-3 & $5.53^{z}$ & ND $^{y}$ \\
SB-4 & $5.7^{z}$ & ND $^{y}$
\end{tabular}

$\overline{{ }^{z}}$ The ratio of average chlorophyll a concentration to average chlorophyll b (chl b) concentration, even though chl b was not detectable in some samples.

${ }^{\mathrm{y}}$ The ratio was not calculable because of undetectable chl b concentrations in all repetitions.

NB, no sunburn; ND, no data; SB-1, sunburn severity 1; SB-2, sunburn severity 2; SB-3, sunburn severity 3; SB-4, severity 4. 

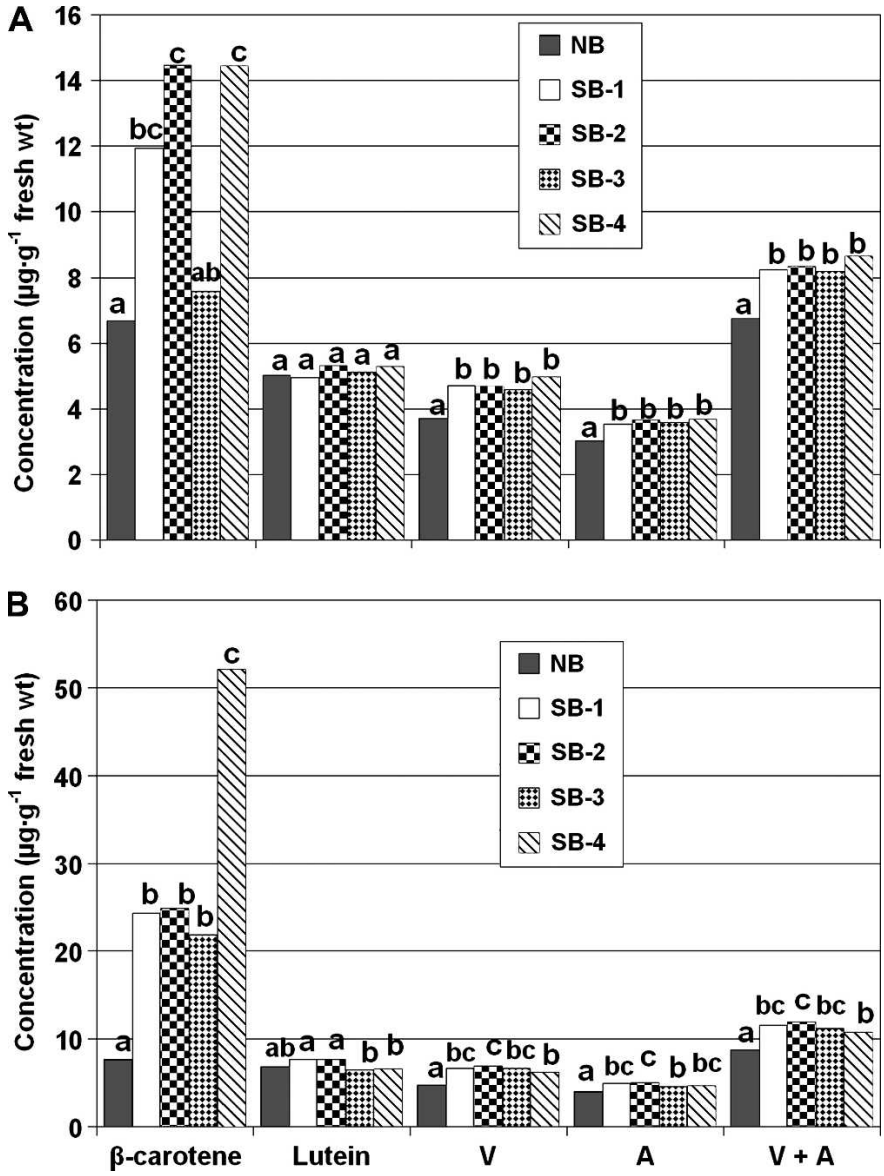

Fig. 3. (A, B) $\beta$-carotene, lutein, violaxanthin (V), and antheraxanthin (A) concentrations in micrograms per gram fresh weight for 'Fuji' apple with peel types NB (no sunburn), SB-1, SB-2, SB-3, and SB-4 (sunburn severity 1-4 respectively) for 2005 (A) and 2006 (B). Within pigment and year, bars with the same letter are not significantly different via LSD at $P \leq 0.05$.

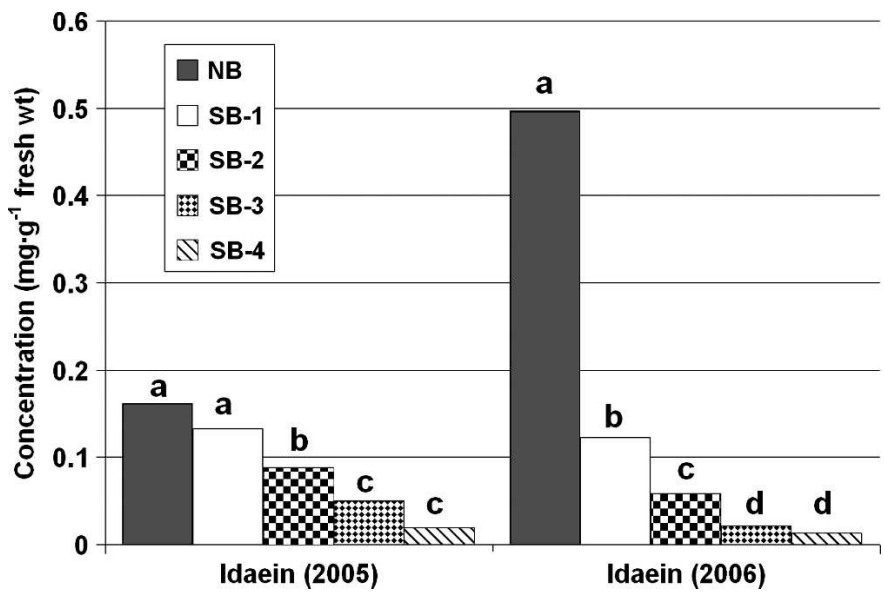

Fig. 4. Idaein concentrations for 'Fuji' apple in 2005 and 2006 with peel types NB (no sunburn), SB-1, SB-2, SB-3, and SB-4 (sunburn severity 1-4 respectively) in milligrams per gram fresh weight. Within year, bars with the same letter are not significantly different via LSD at $P \leq 0.05$.

shown to accumulate at the expense of quercetin 3-arabinofuranoside and, to a lesser extent, quercetin 3-arabinopyranoside.

Chlorogenic acid and epicatechin increased with increased sunburn severity (Fig. 6). Chlorogenic acid in SB-1 was 50\%
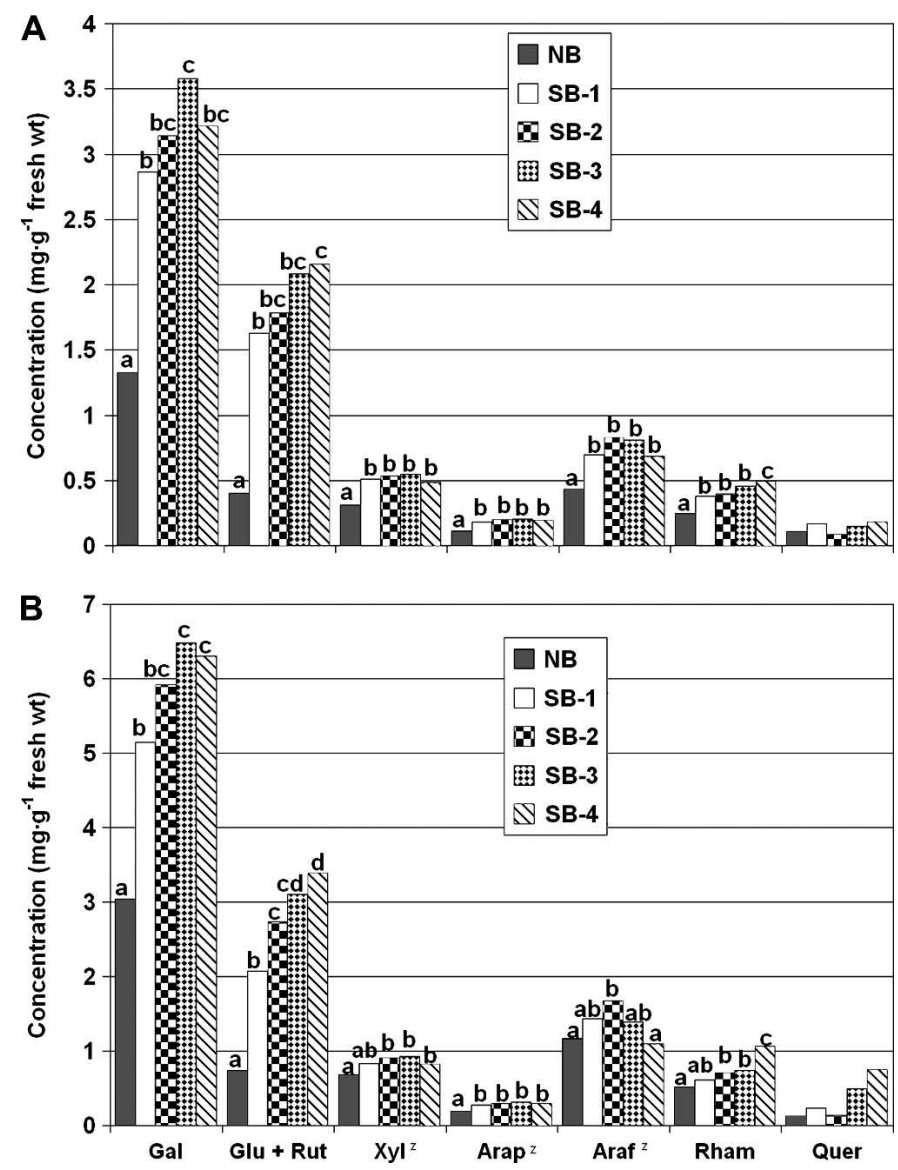

Fig. 5. (A, B) Concentrations of individual quercetin glycosides for 'Fuji' apple in 2005 (A) and 2006 (B) with peel types NB (no sunburn), SB-1, SB-2, SB-3, and SB-4 (sunburn severity 1-4 respectively) measured in milligrams per gram fresh weight. Araf, quercetin 3-arabinofuranoside; Arap, quercetin 3-arabinopyranoside; Gal, quercetin 3-galactoside; Glu + Rut, quercetin 3-glucoside and quercetin 3-rutinoside; Quer, quercetin; Rham, quercetin 3rhamnoside; Xyl, quercetin 3-xyloside. Within compound and year, bars with the same letter are not significantly different via LSD at $P \leq 0.05$. ${ }^{\text {zT }}$ Tentative identification.

and $70 \%$ higher than in NB in 2005 and 2006 respectively. Chlorogenic acid in SB-4 was 99\% and 138\% higher than in SB-3 in 2005 and 2006 respectively. In both years, SB-4 had a higher chlorogenic acid concentration than all other degrees of sunburn. In 2005, SB-1, SB-2, and SB-3 were higher than NB, lower than SB-4, but not different from each other. In 2006, SB-1, SB-3, and SB-4 were higher than NB, but both SB-1 and SB-2 were lower than SB-3 and SB-4, and SB-3 was lower than SB-4.

In 2005, the epicatechin concentration of the NB peel was significantly lower than that of SB-3 and SB-4, but in 2006 NB was significantly lower than all the burned peel types (Fig. 6). In both years, SB-1, SB-2, and SB-3 epicatechin concentrations were not different from each other. In 2005, SB-1 and SB-2 were significantly lower than SB-4, but in 2006 they were not different from SB-4.

The colorimetric data showed general increases in the L* (i.e., getting lighter in color) and the hue angle (i.e., shifting from red to orange) and a general decrease in $\mathrm{C}^{*}$ (i.e., becoming less saturated) as sunburn severity increased (Table 3 ). The exception to this was the 2006 SB-4 peel, which had a lower $\mathrm{L}^{*}$ value than SB-1, and hue angle and $\mathrm{C}^{*}$ values that were 
Table 2. Concentrations of individual quercetin glycosides and quercetin in the final extract.

\begin{tabular}{|c|c|c|c|c|c|c|c|}
\hline $\begin{array}{l}\text { Time after } \\
\text { Extraction } \\
\text { Completion } \\
(\mathrm{min}) \\
\end{array}$ & $\begin{array}{c}\text { Gal } \\
\left(\mathrm{mg} \cdot \mathrm{g}^{-1} \text { fresh wt) }\right.\end{array}$ & $\begin{array}{c}\text { Glu }+ \text { Rut } \\
\left(\mathrm{mg} \cdot \mathrm{g}^{-1} \text { fresh wt }\right)\end{array}$ & $\begin{array}{c}\mathrm{Xyl}^{\mathrm{z}} \\
\left(\mathrm{mg} \cdot \mathrm{g}^{-1} \text { fresh wt) }\right.\end{array}$ & $\begin{array}{c}\operatorname{Arap}^{z} \\
\left(\mathrm{mg} \cdot \mathrm{g}^{-1} \text { fresh wt) }\right.\end{array}$ & $\begin{array}{c}\operatorname{Araf}^{z} \\
\left(\mathrm{mg} \cdot \mathrm{g}^{-1} \text { fresh wt) }\right.\end{array}$ & $\begin{array}{c}\text { Rham } \\
\left(\mathrm{mg} \cdot \mathrm{g}^{-1} \text { fresh wt) }\right.\end{array}$ & $\begin{array}{c}\text { Quer } \\
\left(\mathrm{mg} \cdot \mathrm{g}^{-1} \text { fresh wt) }\right.\end{array}$ \\
\hline 55 & 3.15 & 0.63 & 0.49 & 0.41 & 0.95 & 0.38 & 0.06 \\
\hline 110 & 3.14 & 0.64 & 0.49 & 0.38 & 0.86 & 0.38 & 0.14 \\
\hline 165 & 3.07 & 0.63 & 0.47 & 0.36 & 0.78 & 0.37 & 0.21 \\
\hline 275 & 3.14 & 0.65 & 0.46 & 0.24 & 0.68 & 0.36 & 0.31 \\
\hline 330 & 3.11 & 0.66 & 0.46 & 0.23 & 0.63 & 0.36 & 0.36 \\
\hline 385 & 3.08 & 0.65 & 0.44 & 0.21 & 0.59 & 0.35 & 0.39 \\
\hline 440 & 3.10 & 0.63 & 0.44 & 0.19 & 0.55 & 0.34 & 0.44 \\
\hline 495 & 3.08 & 0.63 & 0.43 & 0.19 & 0.51 & 0.34 & 0.47 \\
\hline
\end{tabular}

${ }^{\mathrm{z}}$ Tentative identification.

Araf, quercetin 3-arabinofuranoside; Arap, quercetin 3-arabinopyranoside; Gal, quercetin 3-galactoside; Glu + Rut, quercetin 3-glucoside and quercetin 3-rutinoside; Xyl, quercetin 3-xyloside; Quer, quercetin; Rham, quercetin 3-rhamnoside.

between the SB-2 and SB-3 values. The hue angle was linearly correlated to the changes in total quercetin glycosides, idaein, and total chlorophyll concentrations (Table 4). The hue angle was also linearly correlated to the total carotenoid concentration in 2006 (Table 4).

\section{Discussion}

The data showed significant concentration changes in pigments that are associated with changes in the degree of sunburn. Results of the chlorophyll and carotenoid analyses are consistent with previous reports on the responses of leaves and apple fruit to increasing solar radiation (Demmig-Adams, 1998; Demmig-Adams and Adams, 1992; Ma and Cheng, 2003, 2004; Thayer and Bjorkman, 1990). These reports compared shaded versus sun-exposed tissues and reported that shaded tissues contained more chlorophyll, less violaxanthin and antheraxanthin, and the same amount of lutein. The increase in the chl a-to-chl b ratio is particularly indicative of a light acclimation response and indicates a reduction in the amount of light harvesting complex II (LHCII), which contains most of the chl b (Green and Durnford, 1996; Kitajima and Hogan, 2003).

We suggest that an upregulation of violaxanthin and antheraxanthin accounts for the higher concentrations of these pigments seen in SB-1 to SB-4 compared with NB. This increase indicates a need for increased nonphotochemical quenching capacity, which suggests that the SB peel types received more irradiation than the NB peel type. However, the relative lack of differences among the SB peel types suggests 1) there is no need for further upregulation or 2) there is a limited capacity of the xanthophyll pigments to upregulate. The stepwise decline of the chlorophylls suggests a need for further upregulation of the xanthophyll cycle, but the data show a limited capacity to upregulate. This limitation could be the result of limited finite resources (i.e., enzymes and precursors) that are needed to synthesize the xanthophylls. Because $\beta$ carotene is an immediate precursor to violaxanthin and antheraxanthin, the fact that it was detected indicates that the limitation is not the result of limited precursors. Not only was $\beta$-carotene found, but it was also found in higher concentration in the burned apples. It is important to note that the percent increase of $\beta$-carotene was greater than violaxanthin and antheraxanthin. This is important because it indicates that more $\beta$-carotene had been produced than was converted to xanthophylls.

The increase in $\beta$-carotene under these stress conditions suggests that it is not being converted to xanthophylls. One possible explanation for this is the deactivation of carotenoid
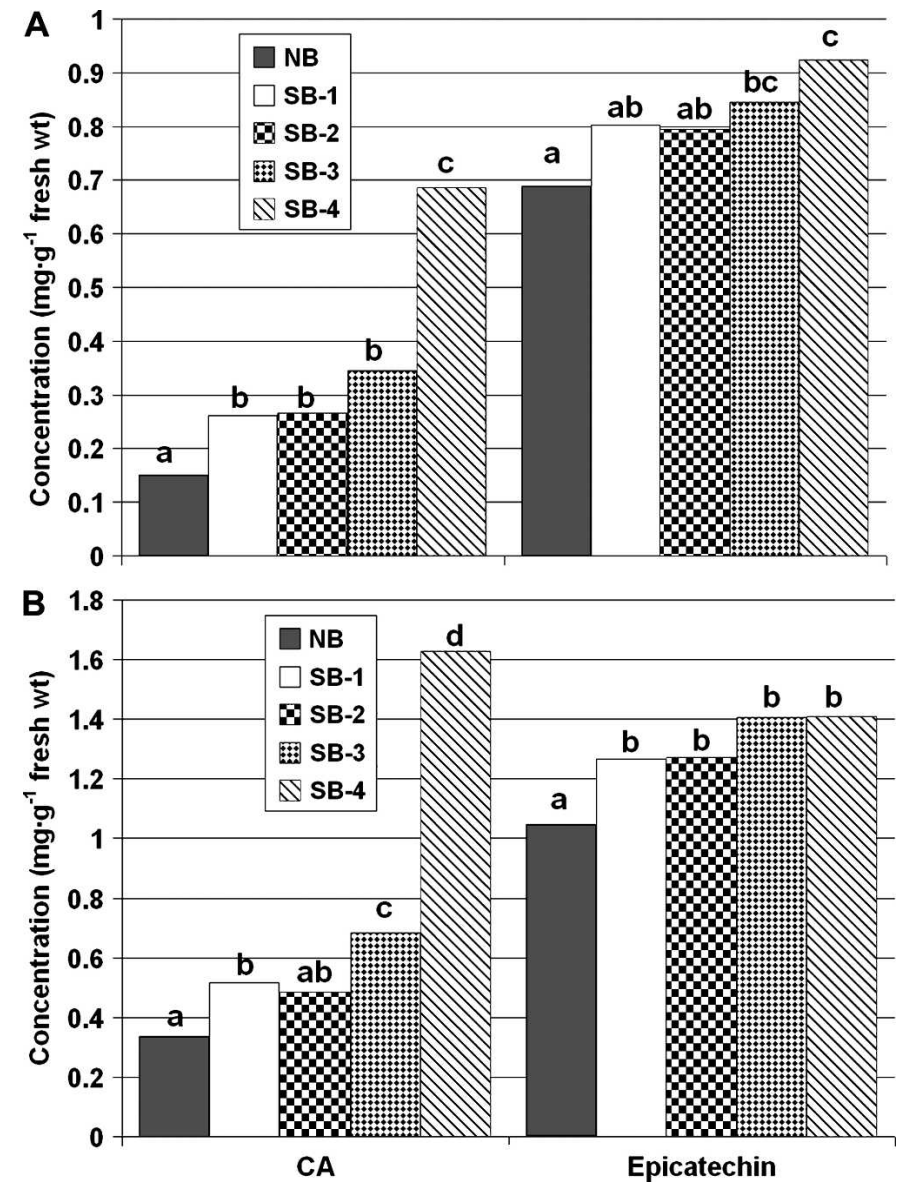

Fig. 6. (A, B) Chlorogenic acid and epicatechin concentrations for 'Fuji' apples in 2005 (A) and 2006 (B) with peel types NB (no sunburn), SB-1, SB-2, SB-3, and SB-4 (sunburn severity 1-4 respectively) in milligrams per grams fresh weight. CA, chlorogenic acid. Within compound and year, bars with the same letter are not significantly different via LSD at $P \leq 0.05$. 
Table 3. Colorimetric data for 2005 and 2006 'Fuji' apples with peel types NB (no sunburn), SB-1, SB-2, SB-3, and SB-4 (sunburn severity $1-4$ respectively).

\begin{tabular}{|c|c|c|c|c|c|c|}
\hline \multirow[b]{2}{*}{ Peel type } & \multicolumn{2}{|c|}{ Lightness factor $^{z}$} & \multicolumn{2}{|c|}{ Hue angle ${ }^{y}$} & \multicolumn{2}{|c|}{ Chroma $^{x}$} \\
\hline & 2005 & 2006 & 2005 & 2006 & 2005 & 2006 \\
\hline$\overline{\mathrm{NB}}$ & $34.9(\mathrm{~d})^{\mathrm{w}}$ & 39.4 (d) & 24.7 (d) & $21.6(d)$ & 33.1 (a) & 34.2 (a) \\
\hline SB-1 & 44.0 (c) & $50.3(\mathrm{c})$ & $39.6(\mathrm{c})$ & $44.8(\mathrm{c})$ & 32.9 (a) & $31.4(b)$ \\
\hline SB-3 & $54.1(\mathrm{a})$ & $61.0(\mathrm{a})$ & $57.2(\mathrm{a})$ & $68.0(\mathrm{a})$ & $25.8(b)$ & 20.7 (d) \\
\hline SB-4 & 52.9 (a) & $48.0(\mathrm{c})$ & 59.7 (a) & $57.1(\mathrm{~b})$ & $24.4(b)$ & $24.6(\mathrm{c})$ \\
\hline
\end{tabular}

Increases in lightness factor indicate that the color is becoming lighter.

${ }^{\mathrm{y}}$ Increases in hue angle indicate a shift from red to orange.

${ }^{x}$ Decreases in chroma indicate reduced color saturation.

${ }^{\text {w} M e a n s ~ w i t h ~ t h e ~ s a m e ~ l e t t e r ~ i n ~ p a r e n t h e s i s ~ a r e ~ n o t ~ s i g n i f i c a n t l y ~ d i f f e r e n t ~ v i a ~ L S D ~ a t ~} P \leq 0.05$.

NB, no sunburn; ND, no data; SB-1, sunburn severity 1; SB-2, sunburn severity 2; SB-3, sunburn severity 3; SB-4, severity 4.

Table 4. Equations for the linear regression of hue angle ${ }^{\mathrm{z}}$ against total chlorophylls, total carotenoids, idaein, and total quercetin glycosides in 'Fuji' apples.

\begin{tabular}{lcccc}
\hline Year & $\begin{array}{c}\text { Slope } \\
(\mathrm{m})\end{array}$ & $\begin{array}{c}\text { y-intercept } \\
(\mathrm{b})\end{array}$ & $\begin{array}{c}R^{2} \\
\text { value }\end{array}$ & $\begin{array}{c}P \\
\text { value }\end{array}$ \\
\hline 2005 & & & & \\
$\quad$ Total chlorophyll & -0.59 & 55.9 & 0.20 & 0.0012 \\
$\quad$ Total carotenoids & 0.43 & 35.52 & 0.05 & 0.12 \\
$\quad$ Idaein & -0.17 & 60.80 & 0.57 & $<0.0001$ \\
$\quad$ Total quercetin & & & & \\
$\quad$ glycosides & 0.0034 & 24.84 & 0.34 & $<0.0001$ \\
2006 & & & & \\
$\quad$ Total chlorophyll & -1.55 & 70.17 & 0.79 & $<0.0001$ \\
$\quad$ Total carotenoids & 0.56 & 25.18 & 0.31 & 0.0042 \\
Idaein & -82.41 & 61.41 & 0.89 & $<0.0001$ \\
Total quercetin & & & & \\
$\quad$ glycosides & 4.84 & -3.40 & 0.73 & $<0.0001$ \\
\hline
\end{tabular}

${ }^{\mathrm{z}}$ Hue angle $=(\mathrm{m} \times$ pigment concentration $)+\mathrm{b}$.

b-ring hydroxylase, which is the enzyme responsible for the conversion of $\beta$-carotene to zeaxanthin. The deactivation of this enzyme would prohibit the formation of violaxanthin and antheraxanthin.

It should be noted that $\beta$-carotene does not solely exist as a precursor to the xanthophylls. It is an accessory pigment that is found in the P680 reaction centers, where it scavenges singlet oxygen and quenches triplet state chlorophyll to protect photosystem II (PSII) (Demmig-Adams et al., 1996). Hence, $\beta$-carotene may be upregulated to help protect PSII under such conditions.

Earlier in the discussion we noted that the observed decline in chlorophyll concentrations was consistent with increased solar radiation. However, under sunburn conditions the fruit surface reaches temperatures in excess of $45^{\circ} \mathrm{C}$ (Schrader et al., 2003b). Reports linking photoxidative stress to lower chlorophyll levels (Merzlyak et al., 1998) and reports that high temperatures alone can reduce photosynthetic efficiency by $53 \%$, thus implying increased photoxidative stress (Torres et al., 2006), raise the question of whether the decline in chlorophyll concentration is solely the result of the increased solar radiation or increased photoxidative stress resulting from high temperatures and high light. Both are possible explanations and, given the conditions under which sunburn occurs, it would be easy to assume the differences in chlorophyll concentration were the result of photoxidative stress. However, this assumption is confounded by the fact that the chl a-to-chl $b$ ratio dramatically increased. The increase in chl a to chl b would require that LHCII be preferentially oxidized over the other chlorophyll-containing protein complexes. Although LHCII is photoxidized, reports indicate that the oxidation results in widespread photoxidation and photobleaching of all the chlorophyll-containing protein complexes (Olszowka et al., 2003; Zucchelli et al., 1988). The change in the chl a-to-chl b ratio indicates that the change is more likely the result of high solar radiation. However, it is not possible to conclude from this experiment whether the changes are the result of varying solar radiation or varying degrees of photoxidative stress.

The phenolic results are particularly intriguing. The most dramatic changes observed were the increases in quercetin glycoside concentrations and the decrease in anthocyanin (idaein) concentration with increased severity of sunburn. Increases in chlorogenic acid, epicatechin, and quercetin glycosides are not surprising given the conditions under which sunburn occurs, because their induction by light is well documented (Bruns et al., 1986; Dixon and Paiva, 1995; Feinbaum et al., 1991; Schmelzer et al., 1988; Spayd et al., 2002; Tattini et al., 2004; Vanderauwera et al., 2005). Although ultraviolet $\mathrm{B}$ radiation seems to be a common source of induction for these compounds, anthocyanins are synergistically induced by red light (Arakawa et al., 1985). The accumulation of these compounds has been linked to protection against radiation in the ultraviolet and visible ranges, with quercetin glycosides protecting against the former and anthocyanins protecting against the latter (Li et al., 1993; Smillie and Hetherington, 1999).

The induction of phenolic compounds by ultraviolet and visible radiation explains the increased chlorogenic acid, epicatechin, and quercetin glycosides concentrations but not the decreased anthocyanin concentrations. Examining the effect of temperature on anthocyanin accumulation can help explain this. Anthocyanins are also induced by cold and inhibited by warm temperatures (Creasy, 1968; Curry, 1997; Dela et al., 2003; Faragher, 1983). This temperature relationship seems to indicate that heat stress would suppress anthocyanin development in apples. However, the literature indicates that this suppression is not permanent (Dela et al., 2003). This should be kept in mind when discussing the temporal separation between sunburn events, normal anthocyanin accumulation, and sample harvest.

Air temperature data (Washington State University, 2007) near the orchards in 2005 and 2006 indicate that ambient 
temperatures in 2005 did not exceed $27.2{ }^{\circ} \mathrm{C}$ from 8 Sept. to 14 Oct. (2005 harvest date). In 2006, the temperature did not exceed $31.1{ }^{\circ} \mathrm{C}$ from 9 Sept. to 18 Oct. (2006 harvest date). In the Pacific northwestern United States, 'Fuji' apples start to develop color in early September and continue to develop color until harvest. It is not known when the sunburn occurred, but given the ambient temperatures it likely did not occur after 9 Sept. either year. This provided $\approx 5$ weeks of sunburnfree conditions for the apples to accumulate anthocyanins during their typical peak anthocyanin accumulation period. The fact that anthocyanin accumulation is depressed indicates a longterm heat stress effect on anthocyanin accumulation in apples.

Decreased anthocyanin concentration is not surprising given the appearance of sunburned apples, but given the current state of knowledge about the biosynthetic pathway of phenolic compounds in apples, it is particularly intriguing that anthocyanin concentrations decreased while epicatechin concentrations increased. Both anthocyanins and epicatechin are synthesized from cyanidin. UDP-glycosyl:flavonoid-3-O-glycosyltransferases (UFGTs) transfer glycosides to cyanidin to form anthocyanins, whereas anthocyanidin reductase (ANR) reduces cyanidin to epicatechin. It may be expected that the difference lies in UFGT. However, UFGT is also required and used to glycosylate quercetin to form the quercetin glycosides. Because quercetin glycosides increased, it appears that UFGTs were present and active. The lack of anthocyanins despite the availability of cyanidin, as demonstrated by the presence of epicatechin, and UFGT has been reported in green leaves (Pfeiffer et al., 2006). Pfeiffer et al. (2006) hypothesized that relative activities of ANR and UFGTs could account for such a dichotomy, and also suggested that temporal and spatial separations in compound formation as well as the transport or the lack of transport of anthocyanins to the vacuole were considerations. The data presented here show increases in epicatechin that pale in comparison with the increases in quercetin glycosides. This suggests that UFGT activities were at least comparable with that of ANR. Because these data represent only one point in time, and ANR and UFGTs were not assayed, it is not known when these compounds were formed or that there were indeed coincidental enzyme activities. Thus, the increases in various compounds may have occurred at different times. Despite this concession, the data do not favor an explanation involving differential enzyme activities, but rather spatial or temporal separation, or issues involving the transport to the vacuole.

As mentioned earlier, quercetin is an artifact of analysis, and is mainly a direct result of the acid hydrolysis of quercetin 3arabinofuranoside and, to a lesser extent, quercetin 3-arabinopyranoside (Table 2). This degradation process explains the significant decrease in quercetin 3-arabinofuranoside concentration observed in 2006 paralleled by a significant increase in quercetin. This degradation is time dependent and can be significant in samples that sit on an autosampler for several hours before being injected into the HPLC system.

Aside from providing an objective measure of the color of the varying degrees of sunburn, the colorimetric data allow probing into the relationship between changes in peel pigments and changes in color. The consistent significant correlation of hue angle to changes in total quercetin glycosides, idaein, and chlorophylls indicate that these pigments are likely contributing to color changes, whereas inconsistent correlations of hue angle to changes in carotenoid concentrations make their involve- ment less certain. These relationships should be interpreted cautiously and are not meant to be used to calculate pigment concentrations based on hue angle, because it is possible to achieve the same hue angle with multiple combinations of pigments (Lancaster et al., 1997). Here they are strictly used to explore the nature of the color change in relationship to changes in the individual pigments.

Although the exact conditions that caused the varying degrees of sunburn browning are not known, sunburn of this type is caused by high FST and high sunlight. It is not unreasonable to speculate that the varying degrees discussed here are the result of differences in FST, irradiance, and/or time of exposure to these factors (i.e., SB-1 was exposed to a lower FST and/or less sunlight than SB-2, 3, or 4). Although it was not the intent of this study to determine the specific conditions at which the varying degrees of sunburn occur, the results support the notion that increased sunburn severity is the result of increased exposure to light or heat stress. Growers can decrease the incidence of sunburn browning by using management practices that keep fruit from attaining a high peel temperature, or that block or reflect harmful ultraviolet radiation. Practices available include overhead evaporative cooling and use of sprayable sunburn protectants such as RAYNOX (Pace International, LLC, Seattle, WA) or particle films such as Surround WP (BASF, Ludwigshafen, Germany). Combining RAYNOX with evaporative cooling was shown to be the most effective treatment for reduction of sunburn (Schrader et al., 2003b)

In conclusion, significant changes in pigment concentrations are associated with changes in sunburn severity, and these changes in concentrations help explain the color changes. The loss of chlorophylls (green) and the decreased anthocyanin (red) accumulation in the sunburned peel allow the colors of the carotenoids (yellow) and quercetin glycosides (yellow, tan) to be more apparent. In addition, the increases in carotenoids and quercetin glycosides cause the discoloration to be even more dramatic.

\section{Literature Cited}

Arakawa, O., Y. Hori, and R. Ogata. 1985. Relative effectiveness and interaction of ultraviolet-B, red and blue light in anthocyanin synthesis of apple fruit. Physiol. Plant. 64:323-327.

Bruns, B., K. Hahlbrock, and E. Schafer. 1986. Fluence dependence of the ultraviolet-light-induced accumulation of chalcone synthase mRNA and effects of blue and far-red light in cultured parsley cells. Planta 169:393-398.

Creasy, L.L. 1968. The role of low temperature in anthocyanin synthesis in 'McIntosh' apples. Proc. Amer. Soc. Hort. Sci. 83: 716-724.

Curry, E.A. 1997. Temperatures for optimum anthocyanin accumulation in apple tissue. J. Hort. Sci. 72:723-729.

Dela, G., E. Or, R. Ovadia, A. Nissim-Levi, D. Weiss, and M. OrenShamir. 2003. Changes in anthocyanin concentration and composition in 'Jaguar' rose flowers due to transient high-temperature conditions. Plant Sci. 164:333-340.

Demmig-Adams, B. 1998. Survey of thermal energy dissipation and pigment composition in sun and shade leaves. Plant Cell Physiol. 39:474-482.

Demmig-Adams, B. and W.W. Adams, III. 1992. Carotenoid composition in sun and shade leaves of plants with different life forms. Plant Cell Environ. 15:411-419.

Demmig-Adams, B., A.M. Gilmore, and W.W. Adams, III. 1996. In vivo functions of carotenoids in plants. FASEB J. 10:403-412. 
Dixon, R.A. and N.L. Paiva. 1995. Stress-induced phenylpropanoid metabolism. Plant Cell 7:1085-1097.

Faragher, J.D. 1983. Temperature regulation of anthocyanin accumulation in apple skin. J. Expt. Bot. 34:1291-1298.

Feinbaum, R.L., G. Storz, and F.M. Ausubel. 1991. High intensity and blue light regulated expression of chimeric chalcone synthase genes in transgenic Arabidopsis thaliana plants. Mol. Gen. Genet. 226:449-456.

Felicetti, D. 2003. Characterization of a third type of apple sunburn caused by sudden exposure of shaded apples to sunlight. Washington State University, Pullman, WA. MS thesis.

Green, B.R. and D.G. Durnford. 1996. The chlorophyll-carotenoid proteins of oxygenic photosynthesis. Annu. Rev. Plant Physiol. Plant Mol. Biol. 47:685-714.

Kitajima, K. and K.P. Hogan. 2003. Increases of chlorophyll a/b ratios during acclimation of tropical woody seedlings to nitrogen limitation and high light. Plant Cell Environ. 26:857-865.

Lancaster, J.E., C.E. Lister, P.F. Reay, and C.M. Triggs. 1997. Influence of pigment composition on skin color in a wide range of fruit and vegetables. J. Amer. Soc. Hort. Sci. 122:594-598.

Li, J., T.M. Ou-Lee, R. Raba, R.G. Amundson, and R.L. Last. 1993. Arabidopsis flavonoid mutants are hypersensitive to UV-B irradiation. Plant Cell 5:171-179.

Ma, F. and L. Cheng. 2003. The sun-exposed peel of apple fruit has higher xanthophyll cycle-dependent thermal dissipation and antioxidants of the ascorbate-glutathione pathway than the shaded peel. Plant Sci. 165:819-827.

Ma, F. and L. Cheng. 2004. Exposure of the shaded side of apple fruit to full sun leads to up-regulation of both the xanthophyll cycle and the ascorbate-glutathione cycle. Plant Sci. 166:1479-1486.

McGuire, R.G. 1992. Reporting of objective color measurements. HortScience 27:1254-1255.

Merzlyak, M.N., A.A. Gitelson, S.I. Pogosyan, L. Lekhimena, and O.B. Chivkunova. 1998. Light-induced pigment degradation in leaves and ripening fruits studied in situ with reflectance spectroscopy. Physiol. Plant. 104:661-667.

Olszowka, D., W. Maksymiec, Z. Krupa, and S. Krawczyk. 2003. Spectral analysis of pigment photobleaching in photosynthetic antenna complex LHCIIb. J. Photochem. Photobiol. B Biol. 70: 21-30.

Pfeiffer, J., C. Kuhnel, J. Brandt, D. Duy, P.A.N. Punyasiri, G. Forkmann, and T.C. Fischer. 2006. Biosynthesis of flavan 3-ols by leucoanthocyanidin 4-reductases and anthocyanidin reductases in leaves of grape (Vitis vinifera L.), apple (Malus $\times$ domestica Borkh.) and other crops. Plant Physiol. Biochem. 44:323-334.

Rudell, D.R., J.P. Mattheis, X. Fan, and J.K. Fellman. 2002. Methyl jasmonate enhances anthocyanin accumulation and modifies production of phenolics and pigments in 'Fuji' apples. J. Amer. Soc. Hort. Sci. 120:435-441.

Schieber, A., P. Hilt, J. Conrad, U. Beifuss, and R. Carle. 2002. Elution order of quercetin glycosides from apple pomace extracts on a new
HPLC stationary phase with hydrophilic endcapping. J. Separation Sci. 25:361-364.

Schmelzer, E., W. Jahnen, and K. Hahlbrock. 1988. In situ localization of light-induced chalcone synthase mRNA, chalcone synthase, and flavonoid end products in epidermal cells of parsley leaves. Proc. Natl. Acad. Sci. USA 85:2989-2993.

Schrader, L.E., J. Sun, D. Felicetti, J. Seo, L. Jedlow, and J. Zhang. 2003a. Stress-induced disorders: Effects on apple fruit quality. Proc. Wash. Tree Fruit Postharvest Conf., Wenatchee, WA, Dec. 2003. 26 Aug. 2007. <http://postharvest.tfrec.wsu.edu/PC2003A.pdf $>$.

Schrader, L.E., J. Zhang, and W.K. Duplaga. 2001. Two types of sunburn in apple caused by high fruit surface (peel) temperature. Plant Health Prog. DOI:10.1094/PHP-2001-1004-01-RS. 26 Aug. 2007. <http://hort.tfrec.wsu.edu/les/Temperature.pdf>.

Schrader, L.E., J. Zhang, and J. Sunday. 2003b. Environmental stresses that cause sunburn of apple. Acta Hort. 618:397-405.

Smillie, R.M. and S.E. Hetherington. 1999. Photoabatement by anthocyanin shields photosynthetic systems from light stress. Photosynthetica 36:451-463.

Smith, T.J. 2007. 2007 Crop protection guide for tree fruits in Washington. 26 Aug. 2007. <http://cru.cahe.wsu.edu/CEPublications/ eb0419/eb0419.pdf>.

Spayd, S.E., J.M. Tarara, D.L. Mee, and J.C. Ferguson. 2002. Separation of sunlight and temperature effects on the composition of Vitis vinifera cv. Merlot berries. Amer. J. Enol. Viticult. 53:171-182.

Tattini, M., C. Galardi, P. Pinelli, R. Massai, D. Remorini, and G. Agati. 2004. Differential accumulation of flavonoids and hydroxycinnamates in leaves of Ligustrum vulgare under excess light and drought stress. New Phytol. 163:547-561.

Thayer, S.S. and O. Bjorkman. 1990. Leaf xanthophyll content and composition in sun and shade determined by HPLC. Photosyn. Res. 23:331-343.

Torres, C.A., P.K. Andrews, and N.M. Davies. 2006. Physiological and biochemical responses of fruit exocarp of tomato (Lycopersicon esculentum Mill.) mutants to natural photo-oxidative conditions. J. Expt. Bot. 57:1933-1947.

Washington State University. 2007. AgWeatherNet. 5 Oct. 2007. $<$ http://agweathernet.prosser.wsu.edu $>$.

Vanderauwera, S., P. Zimmermann, S. Rombauts, S. Vandenabeele, C. Langebartels, W. Gruissem, D. Inze, and F. Van Breusegem. 2005. Genome-wide analysis of hydrogen peroxide-regulated gene expression in arabidopsis reveals a high light-induced transcriptional cluster involved in anthocyanin biosynthesis. Plant Physiol. 139:806-821.

Yamauchi, N. and A.E. Watada. 1991. Regulated chlorophyll degradation in spinach leaves during storage. J. Amer. Soc. Hort. Sci. 116:58-62.

Zucchelli, G., F.M. Garlaschi, and R.C. Jennings. 1988. Spectroscopic analysis of chlorophyll photobleaching in spinach thylakoids, grana and light-harvesting chlorophyll $\mathrm{a} / \mathrm{b}$ protein complex. J. Photochem. Photobiol. B: Biol. 2:490. 